\title{
Hypothyroidism incidence in and around pregnancy: a Danish nationwide study
}

\author{
S L Andersen 1,2, A Carlé ${ }^{1}$, J Olsen ${ }^{3}$ and P Laurberg ${ }^{1,4,+}$ \\ ${ }^{1}$ Departments of Endocrinology, and ${ }^{2} \mathrm{Clinical}$ Biochemistry, Aalborg University Hospital, Aalborg, \\ Denmark, ${ }^{3}$ Department of Clinical Epidemiology, Aarhus University Hospital, Aarhus, Denmark, and \\ ${ }^{4}$ Department of Clinical Medicine, Aalborg University, Aalborg, Denmark \\ ${ }^{\dagger}$ (deceased)
}

Correspondence should be addressed to $S \mathrm{~L}$ Andersen Email stine.a@rn.dk

\begin{abstract}
Objective: Immunological changes in and after a pregnancy may influence the onset of autoimmune diseases. An increased incidence of hyperthyroidism has been observed both in early pregnancy and postpartum, but it remains to be studied if the incidence of hypothyroidism varies in a similar way. Design: Population-based cohort study using Danish nationwide registers.

Method: All women who gave birth to a singleton live-born child in Denmark from 1999 to $2008(n=403958)$ were identified, and data on hospital diagnosis of hypothyroidism and redeemed prescriptions of thyroid hormone were extracted. The overall incidence rate (IR) of hypothyroidism during 1997-2010 and the IR in three-month intervals before, during and after the woman's first pregnancy in the study period were calculated and compared with the IR of hyperthyroidism.

Results: Altogether 5220 women were identified with onset of hypothyroidism from 1997 to 2010 (overall IR 92.3/100 000/year) and 1572 women developed hypothyroidism in the period from 2 years before to 2 years after birth of the first child in the study period. The incidence of hypothyroidism decreased during the pregnancy (incidence rate ratio (IRR) vs overall IR in the rest of the study period: first trimester: 0.89 (95\% Cl: $0.66-1.19)$, second trimester: 0.71 (0.52-0.97), third trimester: $0.29(0.19-0.45))$ and increased after birth with the highest level at 4-6 months postpartum (IRR $3.62(2.85-4.60)$ ).

Conclusion: These are the first population-based data on the incidence of hypothyroidism in and around pregnancy. The incidence declined during pregnancy followed by a sharp increase postpartum. Notably, hypothyroidism as opposed to hyperthyroidism showed no early pregnancy increase.
\end{abstract}

\section{Introduction}

Autoimmune thyroid diseases result from nonphysiological alterations in the immune system that either stimulate the thyroid gland to excessive production of thyroid hormone or damage the thyroid gland and compromise its ability to produce and secrete thyroid hormone (1). Pregnancy is a period of physiological changes in the immune system with a typical immune suppression during the pregnancy followed by an immune rebound after birth of the child (2).
These pregnancy-associated physiological changes in the maternal immune system may influence the onset of an autoimmune thyroid disease (2). One type of autoimmune thyroid disease associated with pregnancy is postpartum thyroiditis that may lead to both transient hyperthyroidism and hypothyroidism (3). However, we previously observed that the incidence of prolonged maternal hyperthyroidism in need of antithyroid drug therapy was high in the postpartum period, and also
두 2016 European Society of Endocrinology Printed in Great Britain
Published by Bioscientifica Ltd. 
in the early pregnancy, which is at variance with other diseases of autoimmune origin (e.g. rheumatoid arthritis and inflammatory bowel disease) (4).

We used data from the Danish nationwide registers to study the incidence of prolonged maternal hypothyroidism in and around pregnancy. We aimed to identify women with persistent, autoimmune hypothyroidism that was diagnosed for the first time in the period from 2 years before to 2 years after the birth of a child and to provide estimates on the incidence in three-month intervals in and around pregnancy. We a priori expected that the postpartum period would be a significant risk factor for the onset of hypothyroidism. This would be in accordance with the 'immune rebound hypothesis' previously proposed by Amino et al. (5) as well as the postpartum peak observed for hyperthyroidism (4) and the previous notion that some women with hypothyroidism apparently caused by postpartum thyroiditis are in need of permanent thyroid hormone replacement therapy (6). A pertinent question was how the incidence of hypothyroidism varied during the pregnancy and how this compared with the variation observed for hyperthyroidism.

\section{Methods}

\section{Study population}

We conducted a population-based cohort study using Danish nationwide registers and studied all women who gave birth to a singleton live-born child in Denmark from 1st January 1999, to 31st December 2008, and had no registration of multiple births. In Denmark, nationwide health data are systematically registered and a unique 10-digit identification number is provided at birth and used in all the registers. Information on all births in Denmark is available in the Danish Medical Birth Register (MBR) (7), and we identified the woman's first live birth in the study period and the 2 year period before and after this event. In addition, the MBR provided information on the woman's age, parity and smoking during the pregnancy. The Statistic Denmark had information on geographical residence, cohabitation and origin. The study was approved by the Danish Data Protection Agency.

\section{Maternal hypothyroidism}

We studied incident hypothyroidism diagnosed in the age of $15-45$ years, which in Denmark predominantly is spontaneous, autoimmune hypothyroidism (8). For the identification of maternal thyroid disease, information was available on hospital diagnosis of the disease, thyroid surgery procedure and redeemed prescriptions of thyroid medication. The Danish National Prescription Register (DNPR) (9) holds nationwide data on all redeemed prescriptions of drugs from Danish pharmacies since 1995 classified according to the Anatomical Therapeutic Chemical classification system (ATC). The Danish National Hospital Register (DNHR) (10) holds nationwide data on all inpatient visits to any Danish hospital since 1977 and all hospital inpatient and outpatient visits since 1995 classified according to the 8th revision of the International Classification of Disease (ICD-8) from 1977 to 1993 and the 10th revision (ICD-10) from 1994.

Women who redeemed prescriptions of antithyroid drugs (ATC H03B) were first identified and classified with hyperthyroidism, as described previously in detail (4). After this, incident cases of prolonged hypothyroidism were defined as women who redeemed a minimum of two prescriptions of thyroid hormone (ATC H03A) in the period from 1st January 1997 to 31st December 2010 and who redeemed prescriptions for a period of more than 2 years and had no hospital diagnosis of congenital or iatrogenic hypothyroidism. In addition, women classified with incident hypothyroidism had no previous redeemed prescription(s) of thyroid hormones, no hospital diagnosis of hypothyroidism (ICD-8: 243.99244.09 and ICD-10 groups: E03 and E89.0) more than three months before the first redeemed prescription of ATD and no previous registration of thyroid surgery (ICD-8: 080.60-082.20 and ICD-10: BAA20-BAA60). The time of onset of hypothyroidism was defined by the date the first prescription of thyroid hormone was redeemed from a Danish pharmacy. Women identified with onset of hypothyroidism in the years 2008-2010 and who redeemed multiple prescriptions of thyroid hormone were included, although treatment beyond 2 years could not be ascertained (end of follow-up in 2010).

\section{Statistical analysis}

The incidence rate (IR) of maternal hypothyroidism from 1997 to 2010 was estimated as the number of women with onset of hypothyroidism divided by the total years of observation time and expressed per 100000 women per year with $95 \%$ confidence interval (95\% CI). The IR of maternal hypothyroidism was subsequently calculated in each three-month interval in and around the woman's first pregnancy in the study period. The incidence rate 
ratio (IRR) with 95\% CI was calculated by comparison with the IR in the rest of the period 1997-2010, using the method described previously in detail (4).

Additional analyses included a comparison with women identified with transient hypothyroidism (treatment for a period of less than 2 years) and sub-analyses of the association to maternal parity. Statistical analyses were performed using STATA version 11 (Statacorp). A level of significance of 5\% was chosen.

\section{Results}

\section{Main analyses}

Altogether 403958 women were included in the study and gave birth to a singleton live-born child in Denmark from 1999 to 2008. Among these, a total of 3679 women had previously been identified with hyperthyroidism. In this study, a total of 5220 women were identified with onset of hypothyroidism from 1997 to 2010 (median age at diagnosis 33.7 years (interquartile range

Table 1 Characteristics of mothers giving birth to a singleton live-born child in Denmark from 1999 to 2008 according to maternal onset of hypothyroidism from 1997 to 2010. Information was obtained at the time of the mother's first birth of a child in the study period.

\begin{tabular}{|c|c|c|c|c|}
\hline & $\begin{array}{r}\text { No inc } \\
\text { hyperthy } \\
\text { or hypoth } \\
1997 \\
\end{array}$ & $\begin{array}{l}\text { ent } \\
\text { idism } \\
\text { oidism } \\
10 \\
\end{array}$ & $\begin{array}{r}\text { Inci } \\
\text { hypoth } \\
1997 \\
\end{array}$ & $\begin{array}{l}\text { t } \\
\text { idism } \\
10\end{array}$ \\
\hline & $n$ & $\%$ & $n$ & $\%$ \\
\hline Mothers & 395065 & & 5220 & \\
\hline Age (years) & & & & \\
\hline$\leq 30$ & 240868 & 61.0 & 2939 & 56.3 \\
\hline$>30$ & 154197 & 39.0 & 2281 & 43.7 \\
\hline Parity* & & & & \\
\hline 1 & 256476 & 64.9 & 3087 & 59.1 \\
\hline$\geq 2$ & 138589 & 35.1 & 2133 & 40.9 \\
\hline Cohabitation & & & & \\
\hline Married & 201317 & 51.0 & 3042 & 58.3 \\
\hline Not married & 193748 & 49.0 & 2178 & 41.7 \\
\hline Origin & & & & \\
\hline Born in Denmark & 347698 & 88.0 & 4443 & 85.1 \\
\hline Not born in Denmark & 47367 & 12.0 & 777 & 14.9 \\
\hline Residence & & & & \\
\hline West Denmark & 208394 & 52.7 & 2623 & 50.3 \\
\hline East Denmark & 186671 & 47.3 & 2597 & 49.7 \\
\hline Smoking in pregnancy & & & & \\
\hline Yes & 77386 & 20.5 & 788 & 15.8 \\
\hline No & 300032 & 79.5 & 4193 & 84.2 \\
\hline
\end{tabular}

*Previous live birth and stillbirth including index pregnancy; ${ }^{\S}$ Reported smoking or smoking cessation in the pregnancy. Mothers with missing value on smoking not included.

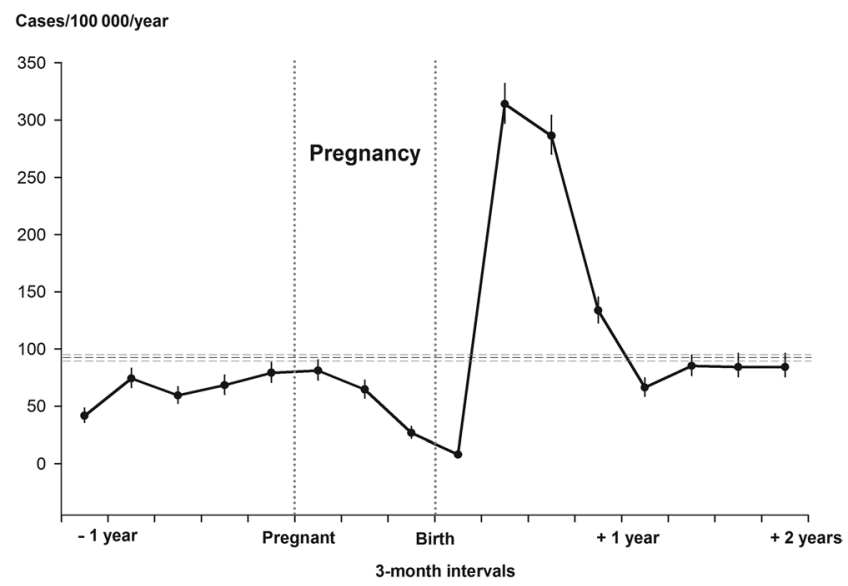

Figure 1

Incidence rates (with 95\% confidence interval) of maternal hypothyroidism in 3-month intervals before, during and after the first pregnancy leading to birth of a live-born child from 1999 to 2008 in a Danish nationwide study of 403958 women. The dashed horizontal lines are the overall incidence rate of maternal hypothyroidism in women aged 15-45years in the study period from 1997 to 2010 (92.3/100 000/year) with 95\% confidence interval.

(IQR): 29.8-37.8 years), median duration of treatment for hypothyroidism in the observation period: 6.0 years (IQR 4.1-8.4 years)), leaving 395065 women with no incident hypothyroidism or hyperthyroidism. All women were studied in relation to their first pregnancy in the study period. Maternal characteristics indicated that women with hypothyroidism were older, less frequently smoking and more often living in East Denmark (Table 1).

The overall IR of maternal hypothyroidism from 1997 to 2010 (horizontal line in Fig. 1) was 92.3/100000/year (95\% CI: 89.9-94.9) compared with that of 65.0/100000/ year for maternal hyperthyroidism. Among cases of incident hypothyroidism, altogether 1572 women had onset of disease in the 4-year period from 2 years before to 4 years after birth of the first child in the study period (median age at diagnosis 30.7 years (IQR: 27.8-34.1 years)). The IR of hypothyroidism in and around pregnancy declined during the pregnancy (Fig. 1) followed by a sharp increase after birth of the child that reached the highest level at 4-6months postpartum (IRR first trimester vs the rest of the study period: 0.89 (95\% CI: 0.66-1.19)), second trimester $(0.71(0.52-0.97))$, third trimester $(0.29$ (0.19-0.45)) and 4-6 months postpartum (3.62 (2.85-4.60)).

The IR of maternal hypothyroidism was subsequently compared with that of hyperthyroidism (Fig. 2). 


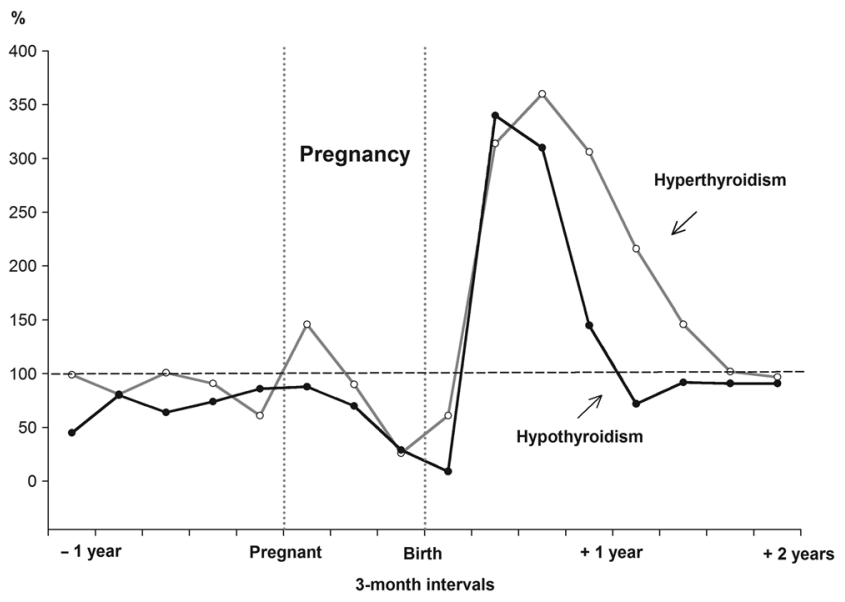

Figure 2

Relative frequency of incident maternal hypothyroidism and hyperthyroidism before, during and after the first pregnancy leading to birth of a live-born child from 1999 to 2008 in a Danish nationwide study of 403958 women. The reference (set to $100 \%$ ) is the overall incidence rate of disease in the study population from 1997 to 2010 (92.3/100000/year for maternal hypothyroidism and 65.0/100000/year for hyperthyroidism).

Both types of thyroid dysfunction showed a decline late in pregnancy, but only hyperthyroidism showed an early pregnancy increase. After birth of the child, both hypothyroidism and hyperthyroidism showed a sharp incidence increase, but the incidence peak was earlier for hypothyroidism and completed within 1 year postpartum (Fig. 2).

\section{Additional analyses}

An increased incidence of hypothyroidism postpartum was observed both among women who had given birth to their first child and also when the studied postpartum period was after the woman's second, third or later child birth. However, the IRR for postpartum onset was higher after a woman's first pregnancy (IRR for onset 4-6 months postpartum vs the rest of the study period: 4.41 (95\% CI: 3.45-5.64)) than after a second or later pregnancy (IRR 2.52 (95\% CI: 2.00-3.18)).

If analyses were restricted to women who only gave birth once during the study period to exclude a possible interference with subsequent pregnancies, these analyses gave a similar incidence pattern in and around pregnancy.

A subgroup of women were not included in the main analyses because their registration of redeemed prescriptions indicated that replacement therapy had

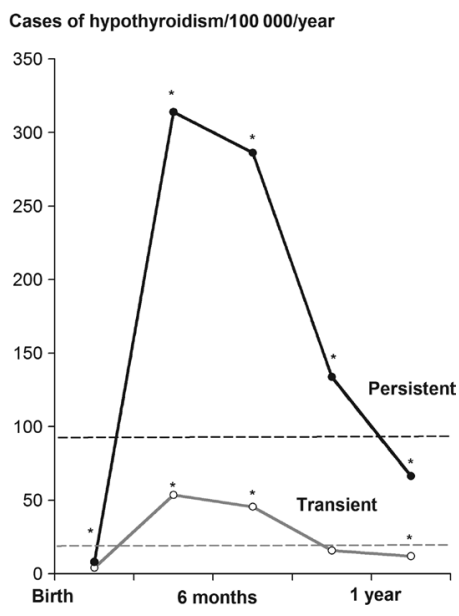

\section{Figure 3}

Incidence rates (IR) of maternal hypothyroidism in 3-month intervals after the birth of a live-born child from 1999 to 2008 in a Danish nationwide study of 403958 women. The dashed horizontal lines are the overall incidence rate of persistent hypothyroidism from 1997 to 2010 (92.3/100000/year) and transient hypothyroidism from 1997 to 2009 (18.3/100 000/year). Women who only redeemed one prescription of thyroid hormone in the last year of the study period (year 2010) were not included due to uncertainty on whether the treatment was transient or persistent. $* 95 \% \mathrm{Cl}$ of the 3-month interval IR and the $95 \% \mathrm{Cl}$ of the overall IR were not overlapping.

been withdrawn within 2 years $(n=1052)$. The overall IR of such transient hypothyroidism that might have been caused by autoimmune thyroiditis from 1997 to 2009 was 18.3/100000/year (95\% CI: 17.5-19.8). Postpartum onset of transient hypothyroidism was less frequently observed than permanent hypothyroidism. However, it showed a similar pattern postpartum with an incidence peak confined to 1 year after birth, reaching the highest level at 4-6 months postpartum (Fig. 3).

\section{Discussion}

\section{Principal findings}

In this Danish population-based study, the incidence of hypothyroidism showed a gradual decline during pregnancy. This variation was followed by an increase in the first year after birth of the child, which peaked at 4-6months postpartum. The incidence pattern was similar to that of hyperthyroidism in late pregnancy and postpartum, but the early pregnancy incidence peak of hyperthyroidism was not observed for hypothyroidism. 


\section{Autoimmune hypothyroidism}

This study aimed to describe the incidence of prolonged maternal hypothyroidism in and around pregnancy. We had no results of thyroid function testing or thyroid autoantibodies, but indicators of persistent hypothyroidism were available from the registration of redeemed prescriptions of thyroid hormone for a period of more than 2 years. The use of the Danish nationwide registrations provided the opportunity to study a large number of women and to estimate the incidence of hypothyroidism in short time intervals in and around pregnancy.

The definition of disease from redeemed prescriptions provided no information on the underlying subtype of hypothyroidism. However, the additional data on hospital diagnosis of disease made it possible in most cases to exclude women with a diagnosis of congenital or iatrogenic hypothyroidism. In Denmark, the predominant type of hypothyroidism is spontaneous, autoimmune hypothyroidism, which in a population-based study with individual review of newly diagnosed cases of overt hypothyroidism was found to be present in $85 \%$ of the cases (8). Postpartum thyroid dysfunction of autoimmune origin was the second most common subtype ( $\sim 5 \%)$, whereas the types of non-autoimmune origin (congenital, iatrogenic, subacute thyroiditis, drug associated) were rare (8).

Thyroid autoantibodies associated with autoimmune hypothyroidism are thyroid-peroxidase antibodies (TPO-Ab) and thyroglobulin antibodies (Tg-Ab), which are directed against the thyroid gland. Such antibodies are prevalent in the population in general (almost 20\% in the Danish population (11)) and considerably more frequent in individuals suffering from autoimmune hypothyroidism (spontaneous or postpartum) $(3,8)$.

\section{Hypothyroidism in pregnancy}

The incidence of maternal hypothyroidism in pregnancy was low with a gradual decline during the pregnancy, which is in agreement with a general suppression of the maternal immune system during pregnancy and the decrease in thyroid autoimmunity (2). TPO-Ab and $\mathrm{Tg}-\mathrm{Ab}$ decrease during pregnancy with a nadir in the third trimester $(12,13,14,15)$.

Maternal hypothyroidism showed no early pregnancy incidence peak. This disparity between hypothyroidism and hyperthyroidism and the similar lack of an early pregnancy peak in other autoimmune diseases (rheumatoid arthritis, inflammatory bowel disease) support the hypothesis that the mechanism for the development of hyperthyroidism in early pregnancy is not related to a general phenomenon in autoimmune diseases (4).

Serum human chorionic gonadotropin (hCG) levels increase in the first trimester of pregnancy and stimulate the thyroid gland. This may lead to the development of gestational hyperthyroidism, which is a non-autoimmune transient disorder. As discussed previously (4), individual review of case registrations indicated that the women identified with incident hyperthyroidism in early pregnancy most likely suffered from Graves' disease. One possible mechanism is that the hCG-mediated increase in the production of thyroid hormone in early pregnancy triggers the onset of Graves' disease in susceptible individuals (16). Likewise, this mechanism may trigger early pregnancy relapse in women with known hyperthyroidism in remission before pregnancy (17).

\section{Hypothyroidism postpartum}

We observed a high incidence of maternal hypothyroidism in the postpartum period. Hypothyroidism incidence peaked earlier than that observed for hyperthyroidism and was confined to 1 year after birth in line with the 'immune rebound hypothesis' (5). In this model (5), the authors suggested that rebound of cellular immunity may exacerbate autoimmune hypothyroidism, whereas the later rebound of humoral immunity may trigger the development of autoimmune hyperthyroidism.

Hypothyroidism in the postpartum period is mainly of autoimmune origin (8). When thyroid dysfunction is detected within 1 year after birth of a child in a woman with no previous diagnosis of thyroid disease, it is classified as postpartum thyroiditis, except if the diagnosis is Graves' disease (3). Postpartum thyroiditis is an autoimmune and common clinical manifestation, which is associated with the presence of TPO-Ab and/or Tg-Ab and can present in various clinical types (e.g. subtle changes in thyroid function that are not clinically detected, isolated thyrotoxicosis or hypothyroidism, or a biphasic course with a thyrotoxic phase followed by hypothyroidism (3)). In a Danish study of 66 TPO-Ab-positive women, 55\% developed postpartum thyroid dysfunction and 50\% of these individuals showed a biphasic course (15).

Thyrotoxicosis in postpartum thyroiditis is typically transient and should not be treated with antithyroid drugs $(18,19)$. As discussed previously (4), the challenge in such patients is to distinguish transient thyrotoxicosis 
in postpartum thyroiditis from the hyperthyroidism of Graves' disease. Considering hypothyroidism, this can be transient in the postpartum period, but some women are in need of permanent thyroid hormone replacement therapy (6). We studied women diagnosed and treated for hypothyroidism for the first time in the postpartum period and who redeemed prescriptions of thyroid hormone for a period of more than 2 years. Thus, the cases we identified were persistent hypothyroidism, but the findings were similar when we subsequently evaluated the postpartum incidence variation in women considered to have transient hypothyroidism.

We observed a high incidence of hypothyroidism in the postpartum period not only among women who had given birth for the first time, but also among multiparous women. In a Danish population-based casecontrol study (20), the number of previous live births and induced abortions were considerable risk factors for the development of autoimmune hypothyroidism after the postpartum period in women diagnosed up to the age of 55 years, but not in postmenopausal women. The proposed mechanism was that repeated pregnancy exposure may accelerate the development of disease in women susceptible to the development of autoimmune hypothyroidism (20). Other studies evaluating the association between parity and the development of autoimmune thyroid disease have shown inconsistent results $(21,22,23,24,25,26$, $27)$. Some studies corroborated an association with parity $(25,26)$, whereas others did not $(21,22,23,24,27)$, but study designs and populations were heterogeneous.

\section{Methodological comments}

The women studied in this register-based design were selected because they had given birth to a singleton liveborn child and women with multiple births were excluded from the study. Thus, the study did not provide data on the incidence of maternal hypothyroidism in and around multiple pregnancies or in case of pregnancy losses.

Women identified with hypothyroidism all redeemed prescriptions of thyroid hormone and had no registration of hyperthyroidism. Thus, women with asymptomatic changes in thyroid function not seen by a doctor and women with no need for medical treatment were not identified. Furthermore, if a woman with a biphasic postpartum thyroiditis (transient hyperthyroidism followed by hypothyroidism) had been diagnosed with hyperthyroidism in a Danish hospital or inappropriately treated with ATD, she would not have been classified with hypothyroidism. These considerations possibly explain why persistent hypothyroidism was more frequent than transient hypothyroidism in this study. 'Onset' of maternal hypothyroidism was defined by the time the first prescription of thyroid hormone was redeemed. The validity of the DNPR is in general considered high (9), and we included a 2-year period after the initiation of the register to exclude prevalent cases.

\section{Conclusion}

The incidence of maternal hypothyroidism in and around a pregnancy was in agreement with the physiological suppression of immune system during the pregnancy followed by an immune rebound after birth. The incidence pattern for hypothyroidism and hyperthyroidism in and around pregnancy was similar in late pregnancy and postpartum but showed a notable difference in the early pregnancy.

\section{Declaration of interest}

The authors declare that there is no conflict of interest that could be perceived as prejudicing the impartiality of the research reported.

\section{Funding}

This research did not receive any specific grant from any funding agency in the public, commercial or not-for-profit sector.

\section{References}

1 Effraimidis G \& Wiersinga WM. Mechanisms in endocrinology: autoimmune thyroid disease: old and new players. European Journal of Endocrinology 2014170 R241-R252. (doi:10.1530/EJE-14-0047)

2 Weetman AP. Immunity, thyroid function and pregnancy: molecular mechanisms. Nature Reviews Endocrinology 20106 311-318. (doi:10.1038/nrendo.2010.46)

3 Stagnaro-Green A. Approach to the patient with postpartum thyroiditis. Journal of Clinical Endocrinology and Metabolism 201297 334-342. (doi:10.1210/jc.2011-2576)

4 Andersen SL, Olsen J, Carle A \& Laurberg P. Hyperthyroidism incidence fluctuates widely in and around pregnancy and is at variance with some other autoimmune diseases: a Danish populationbased study. Journal of Clinical Endocrinology and Metabolism 2015100 1164-1171. (doi:10.1210/jc.2014-3588)

5 Amino N, Tada H \& Hidaka Y. Postpartum autoimmune thyroid syndrome: a model of aggravation of autoimmune disease. Thyroid 19999 705-713. (doi:10.1089/thy.1999.9.705)

6 Premawardhana LD, Parkes AB, Ammari F, John R, Darke C, Adams H \& Lazarus JH. Postpartum thyroiditis and long-term thyroid status: prognostic influence of thyroid peroxidase antibodies and ultrasound echogenicity. Journal of Clinical Endocrinology and Metabolism 200085 71-75. (doi:10.1210/jcem.85.1.6227)

7 Knudsen LB \& Olsen J. The Danish medical birth registry. Danish Medical Bulletin 199845 320-323.

8 Carle A, Laurberg P, Pedersen IB, Knudsen N, Perrild H, Ovesen L, Rasmussen LB \& Jorgensen T. Epidemiology of subtypes of hypothyroidism in Denmark. European Journal of Endocrinology 2006 154 21-28. (doi:10.1530/eje.1.02068) 
9 Kildemoes HW, Sorensen HT \& Hallas J. The Danish national prescription registry. Scandinavian Journal of Public Health 201139 38-41. (doi:10.1177/1403494810394717)

10 Andersen TF, Madsen M, Jorgensen J, Mellemkjoer L \& Olsen JH. The Danish national hospital register. A valuable source of data for modern health sciences. Danish Medical Bulletin 199946 263-268.

11 Pedersen IB, Knudsen N, Jorgensen T, Perrild H, Ovesen L \& Laurberg P. Thyroid peroxidase and thyroglobulin autoantibodies in a large survey of populations with mild and moderate iodine deficiency. Clinical Endocrinology 200358 36-42. (doi:10.1046/j.13652265.2003.01633.x)

12 Amino N, Kuro R, Tanizawa O, Tanaka F, Hayashi C, Kotani K, Kawashima M, Miyai K \& Kumahara Y. Changes of serum anti-thyroid antibodies during and after pregnancy in autoimmune thyroid diseases. Clinical and Experimental Immunology 197831 30-37.

13 Jansson R, Bernander S, Karlsson A, Levin K \& Nilsson G. Autoimmune thyroid dysfunction in the postpartum period. Journal of Clinical Endocrinology and Metabolism 198458 681-687. (doi:10.1210/jcem-58-4-681)

14 Stagnaro-Green A, Roman SH, Cobin RH, el-Harazy E, Wallenstein $\mathrm{S} \&$ Davies TF. A prospective study of lymphocyte-initiated immunosuppression in normal pregnancy: evidence of a T-cell etiology for postpartum thyroid dysfunction. Journal of Clinical Endocrinology and Metabolism $1992 \mathbf{7 4}$ 645-653. (doi:10.1210/jcem.74.3.1740500)

15 Nohr SB, Jorgensen A, Pedersen KM \& Laurberg P. Postpartum thyroid dysfunction in pregnant thyroid peroxidase antibody-positive women living in an area with mild to moderate iodine deficiency: is iodine supplementation safe? Journal of Clinical Endocrinology and Metabolism 200085 3191-3198. (doi:10.1210/jcem.85.9.6799)

16 Laurberg P. Remission of Graves' disease during anti-thyroid drug therapy. Time to reconsider the mechanism? European Journal of Endocrinology 2006155 783-786. (doi:10.1530/eje.1.02295)

17 Amino N, Tanizawa O, Mori H, Iwatani Y, Yamada T, Kurachi K, Kumahara Y \& Miyai K. Aggravation of thyrotoxicosis in early pregnancy and after delivery in Graves' disease. Journal of Clinical Endocrinology and Metabolism 198255 108-112. (doi:10.1210/jcem-551-108)

18 Bahn RS, Burch HB, Cooper DS, Garber JR, Greenlee MC, Klein I, Laurberg P, McDougall IR, Montori VM, Rivkees SA et al. Hyperthyroidism and other causes of thyrotoxicosis: management guidelines of the American thyroid association and American association of clinical endocrinologists. Thyroid 201121 593-646. (doi:10.1089/thy.2010.0417)
19 Stagnaro-Green A, Abalovich M, Alexander E, Azizi F, Mestman J, Negro R, Nixon A, Pearce EN, Soldin OP, Sullivan S et al. Guidelines of the American thyroid association for the diagnosis and management of thyroid disease during pregnancy and postpartum. Thyroid 201121 1081-1125. (doi:10.1089/thy.2011.0087)

20 Carle A, Pedersen IB, Knudsen N, Perrild H, Ovesen L, Rasmussen LB $\&$ Laurberg P. Development of autoimmune overt hypothyroidism is highly associated with live births and induced abortions but only in premenopausal women. Journal of Clinical Endocrinology and Metabolism 201499 2241-2249. (doi:10.1210/jc.2013-4474)

21 Phillips DI, Lazarus JH \& Butland BK. The influence of pregnancy and reproductive span on the occurrence of autoimmune thyroiditis. Clinical Endocrinology 199032 301-306. (doi:10.1111/j.1365-2265.1990.tb00870.x)

22 Walsh JP, Bremner AP, Bulsara MK, O'Leary P, Leedman PJ, Feddema P \& Michelangeli V. Parity and the risk of autoimmune thyroid disease: a community-based study. Journal of Clinical Endocrinology and Metabolism 200590 5309-5312. (doi:10.1210/jc.2005-0771)

23 Bulow Pedersen I, Laurberg P, Knudsen N, Jorgensen T, Perrild H, Ovesen L \& Rasmussen LB. Lack of association between thyroid autoantibodies and parity in a population study argues against microchimerism as a trigger of thyroid autoimmunity. European Journal of Endocrinology 2006154 39-45. (doi:10.1530/eje.1.02070)

24 Strieder TG, Tijssen JG, Wenzel BE, Endert E \& Wiersinga WM. Prediction of progression to overt hypothyroidism or hyperthyroidism in female relatives of patients with autoimmune thyroid disease using the Thyroid Events Amsterdam (THEA) score. Archives of Internal Medicine 2008168 1657-1663. (doi:10.1001/ archinte.168.15.1657)

25 Friedrich N, Schwarz S, Thonack J, John U, Wallaschofski H \& Volzke H. Association between parity and autoimmune thyroiditis in a general female population. Autoimmunity $2008 \mathbf{4 1} 174-180$. (doi:10.1080/08916930701777629)

26 Greer LG, Casey BM, Halvorson LM, Spong CY, McIntire DD \& Cunningham FG. Antithyroid antibodies and parity: further evidence for microchimerism in autoimmune thyroid disease. American Journal of Obstetrics and Gynecology 2011205 471.e1-471.e4. (doi:10.1016/j. ajog.2011.06.060)

27 Bjergved L, Carle A, Jorgensen T, Perrild H, Laurberg P, Krejbjerg A, Ovesen L, Bulow Pedersen I, Rasmussen LB \& Knudsen N. Parity and 11-year serum thyrotropin and thyroid autoantibody change: a longitudinal population-based study. Thyroid 201626 203-211. (doi:10.1089/thy.2014.0279)

Received 25 May 2016

Revised version received 28 July 2016

Accepted 10 August 2016 